\title{
ON THE USE OF CESÃRO MEANS IN DETERMINING CRITERIA FOR FOURIER CONSTANTS*
}

BY C. N. MOORE

1. Introduction. In the portion of Professor Fejér's address dealing with Fourier series, the positiveness of certain linear operations played a central role. These linear operations were those that arise in forming for a series the partial Cesàro means of various integral orders.

The positiveness of the linear functional operation in the case of a wide class of Fourier series resulted from the positiveness in the case of certain very simple trigonometric series. One such series is the series

(1) $\frac{1}{2}+\cos \theta+\cos 2 \theta+\cos 3 \theta+\cdots+\cos n \theta+\cdots$,

whose behavior is of fundamental importance in studying the convergence or summability of the general Fourier series. For the series (1) the partial Cesàro mean of the first order takes the form

$$
\frac{1}{2 n} \cdot \frac{\sin ^{2}(n \theta / 2)}{\sin ^{2}(\theta / 2)}
$$$$
(\theta \neq 2 m \pi),
$$

and the value $n / 2$ for the excepted values of $\theta$, thus being obviously non-negative for all values of $\theta$.

For many questions involving Fourier series further light is shed on the behavior of the series by considering the Cesàro means of non-integral order introduced by Knopp, M. Riesz; and Chapman. These means may be defined as follows. We set

$$
A_{0}^{(k)}=1, A_{n}^{(k)}=\frac{(k+1) \cdots(k+n)}{n !}, \quad(n=1,2, \cdots),
$$

so that

$$
(1-z)^{-(k+1)}=\sum_{n=0}^{\infty} A_{n}^{(k)} z^{n}, \quad(|z|<1),
$$

* Presented to the Society and Section A of the American Association for the Advancement of Science, by invitation, June 21, 1933, on the occasion of an address by Professor L. Fejér. 
the $A$ 's thus being the coefficients in a certain binomial expansion. For any series $\sum u_{n}$, the $n$th Cesàro mean of order $k$ is defined as

$$
\sigma_{n}^{(k)}=\frac{\sum_{\lambda=0}^{\lambda=n} A_{n-\lambda}^{(k-1)} s_{\lambda}}{A_{n}^{(k)}}=\frac{S_{n}^{(k)}}{A_{n}^{(k)}},
$$

and is readily seen to be a weighted mean of the partial sums $s_{0}, s_{1}, s_{2}, \cdots, s_{n}, \cdots$ of the corresponding series. These definitions may be used for any value of $k$ not equal to a negative integer and include the positive, integral means as special cases. We consider them here for real values of $k \geqq 0$.

If we apply the linear functional operation defined by (4) to the partial sums of the series (1) for values of $k$ in the interval $(0<k<1)$, we can no longer assert that the resulting expression is everywhere non-negative, ${ }^{*}$ as in the case $k=1$. However, if we represent this expression by $\sigma_{n}{ }^{(k)}(\theta)$, we find that it has the important property, in common with the expression (2), that

$$
\int_{0}^{\pi}\left|\sigma_{n}^{(k)}(\theta)\right| d \theta
$$

remains bounded for all $n$. We may speak of this property as that of being bounded in the mean for the interval $(0, \pi)$. In view of the evenness and periodicity of the expression $\sigma_{n}^{\left({ }^{(k)}\right.}(\theta)$, it follows readily that it is bounded in the mean for any finite interval.

The ordinary partial sums of the series (1) do not have the property of being bounded in the mean for the interval $(0, \pi)$, since the set of constants

$$
c_{n}=\int_{0}^{\pi}\left|\frac{\sin \left(n+\frac{1}{2}\right) t}{\sin \frac{1}{2} t}\right| d t,
$$

obtained by integrating the absolute value of the partial sums of the series over the interval $(0, \pi)$, become infinite with $n$ approximately as the logarithm of $n$. This property of the constants (6) was demonstrated in 1910 by Fejér, $\uparrow$ who named the

* See Gronwall, On the Cesdro sums of Fourier's and Laplace's series, Annals of Mathematics, (2), vol. 32 (1931), pp. 53-59. See in particular \$2.

$\dagger$ Fejér, Lebesguesche Konstanten und divergente Fourierreihen, Journal für Mathematik, vol. 138 (1910), pp. 22-53. 
set of constants obtained by multiplying (6) by $2 / \pi$, Lebesgue constants. The fact that the set of Lebesgue constants is not bounded is inherently connected with the fact that the Fourier development of certain continuous functions diverges.

The boundedness in the mean of the $\sigma_{n}{ }^{(k)}(\theta)$ for an interval including the origin is a determining factor in the efficacy of summation $(C k)$, when applied to the Fourier series of an $L$-integrable function.* It may also be utilized to derive certain criteria for deciding if a given set of constants are the Fourier constants of an $L$-integrable function, as will be shown in the ensuing discussion.

2. Criteria for Fourier Constants. The criteria in question involve differences of integral or non-integral order formed from an infinite set of constants. We define these as follows, for any set of constants $a_{0}, a_{1}, a_{2}, \cdots, a_{n}, \cdots$ :

$$
\Delta^{k+1} a_{n}=a_{n}-(k+1) a_{n+1}+\frac{(k+1) k}{1 \cdot 2} a_{n+2}-\cdots .
$$

For a non-integral value of $k$ these differences are the limit as $p \rightarrow \infty$, provided this limit exists, of the expressions

$$
\begin{aligned}
& \Delta_{p}^{k+1} a_{n}=a_{n}-(k+1) a_{n+1}+\cdots \\
& \quad+(-1)^{p-n} \frac{(k+1) k \cdots(k-p+n+2)}{1 \cdot 2 \cdots(p-n)} a_{p},(n<p), \\
& \Delta_{p}^{k+1} a_{p}=a_{p} .
\end{aligned}
$$

If for a series $\Sigma u_{n}, S_{n}^{(k)}$ is defined as in (4), we have the identity

$$
\sum_{n=0}^{n=p} u_{n} a_{n}=\sum_{n=0}^{n=p} S_{n}^{(k)} \Delta_{p}^{k+1} a_{n}
$$

by means of a transformation analogous to the well known Abel transformation of a corresponding summation, where the partial sums of the $u$ 's and the first differences of the $a$ 's are used.

* See the discussion of summability in the following papers: Chapman, $O n$ non-integral orders of summability of series and integrals, Proceedings of the London Mathematical Society, vol. 9 (1911), pp. 369-409, in particular \$20; Gronwall, On the summability of Fourier's series, this Bulletin, vol. 20 (1913-14), pp. 139-146. 
For any series $\sum u_{n}$ for which $S_{n}{ }^{(k)}$ remains bounded, we may, if the $a_{n}$ 's satisfy the conditions*

$$
\lim _{n \rightarrow \infty} a_{n}=0, \quad \sum_{n=0}^{\infty} n^{k}\left|\Delta^{k+1} a_{n}\right|<\infty, \quad(k>0),
$$

allow $p$ to become infinite in the above identity, since the expression on the right hand side approaches a limit by virtue of the restrictions imposed. $\dagger$ We thus obtain the result

$$
\lim _{p \rightarrow \infty} \sum_{n=0}^{n=p} S_{n}^{(k)} \Delta_{p}^{k+1} a_{n}=\sum_{n=0}^{\infty} S_{n}^{(k)} \Delta^{k+1} a_{n}=\sum_{n=0}^{\infty} u_{n} a_{n}
$$

We are now ready to state our criteria in the form of a theorem.

THEOREM. If the set of constants $a_{0}, a_{1}, a_{2}, \cdots, a_{n}, \cdots$ satisfy the conditions (10), then the series

(12) $\frac{1}{2} a_{0}+a_{1} \cos \theta+a_{2} \cos 2 \theta+\cdots+a_{n} \cos n \theta+\cdots$

will converge in the open interval $(0<\theta \leqq \pi)$, and will represent there an L-integrable function whose Fourier cosine development is given by (12).

This theorem follows readily from the preceding discussion and the fact that for the trigonometric series (1) the expression $\sigma_{n}{ }^{(k)}(\theta)$ is bounded in the mean in the interval $(0, \pi)$. Because of the conditions on the $a$ 's and the boundedness of $S_{n}{ }^{(k)}(\theta)$ for the series (1) in the interval $0<\delta \leqq \theta \leqq \pi$, we may infer the convergence of the series (12) in that interval to a function

$$
f(\theta)=\frac{1}{2} a_{0}+a_{1} \cos \theta+a_{2} \cos 2 \theta+\cdots=\sum_{n=0}^{\infty} S_{n}^{(k)}(\theta) \Delta^{k+1} a_{n} .
$$

The series on the right hand side of (13) converges absolutely and uniformly in the interval $0<\delta \leqq \theta \leqq \pi$ and can therefore be integrated term by term in that interval. Thus we have

$$
\text { (14) } \int_{\delta}^{\pi}|f(\theta)| d \theta \leqq \sum_{n=0}^{\infty}\left(\int_{\delta}^{\pi}\left|\frac{S_{n}{ }^{(k)}(\theta)}{A_{n}{ }^{(k)}}\right| d \theta\right) \frac{A_{n}^{(k)}}{n^{k}} n^{k}\left|\Delta^{k+1} a_{n}\right| \text {. }
$$

* The existence of the differences in the second condition follows from the first condition.

† See Chapman, loc. cit., $\$ 15$. 
As $\delta$ approaches zero, the right hand side of (14) approaches a definite limit in view of conditions (10) and the boundedness in the mean of $\sigma_{n}{ }^{(k)}(\theta)$ in the interval $(0, \pi)$. Thus the left hand side does also, and the existence of the resulting integral shows that the function $f(\theta)$ is an $L$-integrable function whose Fourier cosine development is given by (12).

3. Relationship to Criteria Previously Found. The second condition in (10) involves a parameter $k$, and thus we have different criteria for different positive values of $k$. If we set $k=1$ in (10), the resulting conditions constitute a criterion due to Kolmogoroff.* It can be shown that the scope of our theorem increases as $k$ diminishes. Thus any of our criteria for a positive value of $k<1$ includes Kolmogoroff's theorem. Furthermore, there is no best criterion of this type, and we have a sliding scale of tests analogous to the logarithmic scale in testing the convergence of a series of positive terms.

For the proof of these facts we need to show that if the differences $\Delta^{k+1} a_{n}$ satisfy the second condition of (10) for a positive value $r$ of $k$, they will satisfy it for any value of $k$ in the interval $0<k<r$. If we set $\rho=r-k$, it is readily seen that we have the formal identity

$$
\Delta^{k+1} a_{n}=\Delta^{-\rho}\left(\Delta^{r+1} a_{n}\right) .
$$

The validity of the infinite process involved in this identity may be established by expressing the right hand side in the form of a double series as follows:

$$
\begin{aligned}
& a_{n}-(r+1) a_{n+1}+\frac{(r+1) r}{1 \cdot 2} a_{n+2}-\ldots \\
& +0+\rho a_{n+1} \quad-\rho(r+1) a_{n+2}+\ldots \\
& +0+0 \quad+\frac{\rho(\rho+1)}{1 \cdot 2} a_{n+2}-\ldots \\
& +. . . . . . . . . . . . . . .
\end{aligned}
$$

If we form the sum, $s_{p q}$, of the $p q$ terms taken from the first $p$ rows and the first $q$ columns of this series, the convergence of

* Kolmogoroff, Sur l'ordre de grandeur des coefficients de la série de FourierLebesgue, Bulletin de l'Académie Polonaise, (A), 1923, pp. 83-86. 
$s_{p q}$ as $p$ and $q$ become infinite in such a manner that $q \leqq p$ follows from the existence of the left hand side of (15). For the case $q>p$, the additional terms included are seen to be negligible from the boundedness of the $a$ 's and the order of magnitude of the binomial coefficients involved. Thus (16) converges as a double series. Furthermore, the individual columns converge since their terms are all zero from a certain point on, and the individual rows converge as a result of the existence of the differences $\Delta^{r+1} a_{n}$. From a theorem due to Pringsheim* it now follows that the double series converges when summed by columns or rows, and that the sum by columns is equal to the sum by rows, which establishes the validity of (15).

We are now ready to show that the convergence of $\sum n^{r}\left|\Delta^{r+1} a_{n}\right|$ implies the convergence of $\sum n^{k}\left|\Delta^{k+1} a_{n}\right|$, whenever $\dagger 0 \leqq k<r$. From (15), taking account of the fact that the right-hand side is the sum by rows of (16), we have the inequality

$$
\left|\Delta^{k+1} a_{n}\right|<\left|\Delta^{r+1} a_{n}\right|+\sum_{p=1}^{\infty} K p^{\rho-1}\left|\Delta^{r+1} a_{n+p}\right|,
$$

Hence, if we set

( $K$ a positive constant).

$$
\left|\Delta^{r+1} a_{n}\right|=\epsilon_{n} / n^{r},
$$

we obtain readily the further inequality

$$
\begin{aligned}
\sum_{n=0}^{\infty} n^{k}\left|\Delta^{k+1} a_{n}\right|<\sum_{n=0}^{\infty} n^{k}\left|\Delta^{r+1} a_{n}\right| & \\
& +\sum_{n=0}^{\infty} n^{k}\left(\sum_{p=1}^{\infty} \frac{K p^{\rho-1}}{(n+p)^{r}} \epsilon_{n+p}\right) .
\end{aligned}
$$

The single series in the first term of the right hand side of (19) is obviously convergent, $k$ being less than $r$. Since, in view of our hypothesis, the $\epsilon_{n}$ defined by (18) is the general term of a convergent series, it will follow that the double series in the second term on the right hand side of (19) is convergent if, when we sum the expression

$$
n^{k} p^{\rho-1} /(n+p)^{r}
$$

* Bromwich, Theory of Infinite Series, 1st or 2d edition, $\$ 30$.

$\dagger$ For our immediate purpose we do not need to include the case $k=0$, but the more general result follows from the same argument. 
over all values of $n$ and $p$ for which $n+p=m$, the result is bounded for all $m$. This summation can be written in the form

$$
\frac{1}{m^{r}} \sum_{p=1}^{p=m} p^{\rho-1}(m-p)^{k}=\sum_{p=1}^{p=m}\left(\frac{p}{m}\right)^{\rho-1}\left(1-\frac{p}{m}\right)^{k} \frac{1}{m} \text {. }
$$

For large values of $m$ the summation on the right hand side of (21) approximates to the value of the integral

$$
\int_{0}^{1} x^{\rho-1}(1-x)^{k} d x
$$

and is therefore bounded for all $m$, as we wished to show.

We have proved that the convergence of $\sum n^{r}\left|\Delta^{r+1} a_{n}\right|$ implies the convergence of $\sum n^{k}\left|\Delta^{k+1} a_{n}\right|$, whenever $r>k \geqq 0$. That the converse is not true may be readily seen by considering the $a$ 's defined by

(22) $a_{0}=0, \lim _{n \rightarrow \infty} a_{n}=0 ; \quad a_{2 n-1}-a_{2 n}=0, \quad a_{2 n}-a_{2 n+1}=\frac{1}{n^{2}}$,

$(n=1,2, \cdots)$. This set of $a$ 's satisfies the second condition in (10) for any $k$ such that $0 \leqq k<1$, but not for $k=1$.

The question naturally arises as to why we can not take the $k$ in (10) equal to zero and thus get a more inclusive theorem than that obtained for values of $k>0$. It is readily seen, however, that if we allow the $k$ to take on the value zero, the argument of our theorem is no longer valid, since the ordinary partial sums of the series (1) are not bounded in the mean for the interval $(0, \pi)$ but become logarithmically infinite in the mean with $n$, as pointed out before. A modification of (10) to meet this difficulty gives us a criterion due to Szidon,*

$$
\lim _{n \rightarrow \infty} a_{n}=0, \quad \sum_{n=1}^{\infty} \log n\left|\Delta a_{n}\right|<\infty .
$$

This latter criterion is obviously closely related to the criteria of the present paper, but it does not include them nor is it included by them.

The University of Cincinnati

* Szidon, Reihentheoretische Sätze und ihre Anwendungen in der Theorie der Fourierschen Reihen, Mathematische Zeitschrift, vol. 10 (1921), pp. 121-127. 